\title{
Luminosity function of clusters of galaxies
}

\author{
M. Paolillo ${ }^{1, \star}$, S. Andreon ${ }^{1}$, G. Longo ${ }^{1}$, E. Puddu ${ }^{1}$, R. R. Gal ${ }^{3}$, R. Scaramella ${ }^{2}$, \\ S. G. Djorgovski ${ }^{3}$, and R. de Carvalho ${ }^{4}$ \\ 1 Osservatorio Astronomico di Capodimonte, via Moiariello 16, 80131 Napoli, Italy \\ 2 Osservatorio Astronomico di Monte Porzio, via Frascati 33, 00044 Roma, Italy \\ 3 Department of Astronomy, Caltech, USA \\ 4 Observatório Nacional, Rua General José Cristino 77, 20921 - 400 Rio de Janeiro, Brazil
}

Received 20 September 2000 / Accepted 5 December 2000

\begin{abstract}
The composite galaxy luminosity function (hereafter LF) of 39 Abell clusters of galaxies is derived by computing the statistical excess of galaxy counts in the cluster direction with respect to control fields. Due to the wide field coverage of the digitised POSS-II plates, we can measure field counts around each cluster in a fully homogeneous way. Furthermore, the availability of virtually unlimited sky coverage allows us to directly compute the LF errors without having to rely on the estimated variance of the background. The wide field coverage also allows us to derive the LF of the whole cluster, including galaxies located in the cluster outskirts. The global composite LF has a slope $\alpha \sim-1.1 \pm 0.2$ with minor variations from blue to red filters, and $M^{*} \sim$ $-21.7,-22.2,-22.4 \mathrm{mag}\left(H_{0}=50 \mathrm{~km} \mathrm{~s}^{-1} \mathrm{Mpc}^{-1}\right)$ in $g, r$ and $i$ filters, respectively (errors are detailed in the text). These results are in quite good agreement with several previous determinations and in particular with the LF determined for the inner region of a largely overlapping set of clusters, but derived making use of a completely different method for background subtraction. The similarity of the two LFs suggests the existence of minor differences between the LF in the cluster outskirts and in the central region, or a negligible contribution of galaxies in the cluster outskirts to the global LF.
\end{abstract}

Key words. galaxies: clusters: general - galaxies: luminosity function - galaxies: evolution

\section{Introduction}

The galaxy luminosity function (hereafter LF) measures the relative frequency of galaxies as a function of luminosity per unit co-moving volume. Thus, the LF is the zero-order statistic of galaxy samples and provides the natural weighting of most statistical quantities. For instance, the luminosity evolution is often inferred by the variation with redshift of the LF; the metal production rate is the integral of the luminosity weighted against the LF; the fraction of blue galaxies, crucial for the ButcherOemler effect (Butcher \& Oemler 1984), is given by the ratio between the color distribution, averaged over the LF, and the total number of galaxies (i.e. the integral of the LF). The LF is, therefore, central to many cosmological issues (Binggeli et al. 1988; Koo \& Kron 1992; Ostriker 1993).

The determination of the cluster LF is observationally less expensive than the analogous determination of the field LF. In fact, the cluster LF can be determined

Send offprint requests to: G. Longo,

e-mail: longo@na.astro.it

* Università di Palermo, D.S.F.A., Sez.di Astronomia P.zza del Parlamento 1, 90134 Palermo, Italy. as the statistical excess of galaxies along the cluster line of sight, with respect to the control field direction, due to the fact that clusters appear as overdensities with respect to the intracluster field. Therefore we do not need to know the redshift of each cluster member but only the mean cluster redshift, provided that we treat the sampled volume as a free parameter. This approach assumes implicitly that the background contribution along the cluster line of sight is equal to the "average" background, a hypothesis that a non-zero correlation function for galaxies shows to be only approximate: there are galaxies near the cluster line of sight, but not belonging to the cluster itself, in excess of the value expected by assuming a uniform "average" galaxy density. In other words, it happens very often that a nearby group, cluster or supercluster contaminates the control field counts or the cluster counts thus affecting the determination of the cluster LF. This problem is even more relevant when sampling the cluster outskirts, where galaxy evolution probably occurs (van Dokkum et al. 1998) since i) the low galaxy density of these regions is affected by even a few contaminants, and ii) the large observing area makes more probable the presence of a contaminating group. Recently, Huang et al. (1997) found an expression for estimating the error 
introduced by a non zero correlation function. This expression however, inflates errors as a consequence of the fact that the statistics is not simply Poissonian, and does not try to correct field counts to the value expected once the contribution due to other prospectically near overdensities is taken into account.

From an observational point of view, a proper determination of the LF with small field of view imagers and in presence of a non-zero correlation function is very time consuming since several fields all around the cluster need to be observed to estimate the field counts along the clusters line of sight. Therefore, in order to save precious telescope time, very often the field counts are taken from the literature (and usually concern a specific region of the sky which is often completely unrelated to the cluster line of sight) or only a few (usually one, except Bernstein et al. 1995) comparison fields at fairly different right ascensions are adopted. The alternative route is to recognize cluster membership individually, for instance on morphological grounds as Binggeli et al. (1985) did for the Virgo cluster, or by means of galaxy colors, as in Garilli et al. (1999, hereafter GMA99).

Wide-field imagers, such as Schmidt plates or large CCD mosaics, allow one instead to sample lines of sight all around the cluster, and accurately determine the field properties along the cluster line of sight (cf. Valotto et al. 1997).

Our group is currently exploiting the Digitized Palomar Sky Survey (DPOSS) and the resulting Palomar-Norris Sky Catalog (PNSC) in the context of the CRoNaRio collaboration (Caltech-Roma-Napoli-Rio) (Djorgovski et al. 1999). Due to the good photometric quality of the data and the wide sky coverage of DPOSS data, the survey is particularly tailored to explore the actual background contribution to the determination of the cluster LF.

This paper is organised as follow: in Sect. 2 we briefly describe the main characteristics of the data and we present the cluster sample. Section 3 deals with most technical problems related to the determination of the individual LF of clusters. Section 4 presents the results of this work and a comparison with literature results. Conclusions are summarised in Sect. 5. We adopt $H_{0}=50 \mathrm{~km} \mathrm{~s}^{-1} \mathrm{Mpc}^{-1}$ and $q_{0}=0.5$.

\section{The data and the sample}

The data used in this paper were extracted from the DPOSS frames taken in the photographic $J, F$ and $N$ bands (Reid et al. 1991). Weir et al. (1995c) describe the characteristics of the SKICAT package, which performs the plate linearization and the object detection and classification (based on a classification tree, see Weir et al. 1995a).

SKICAT measures four different magnitudes for each object detected on the plates, among which the FOCAS (Jarvis \& Tyson 1981) total magnitude, obtained by dilatation of the detection isophote in all directions until

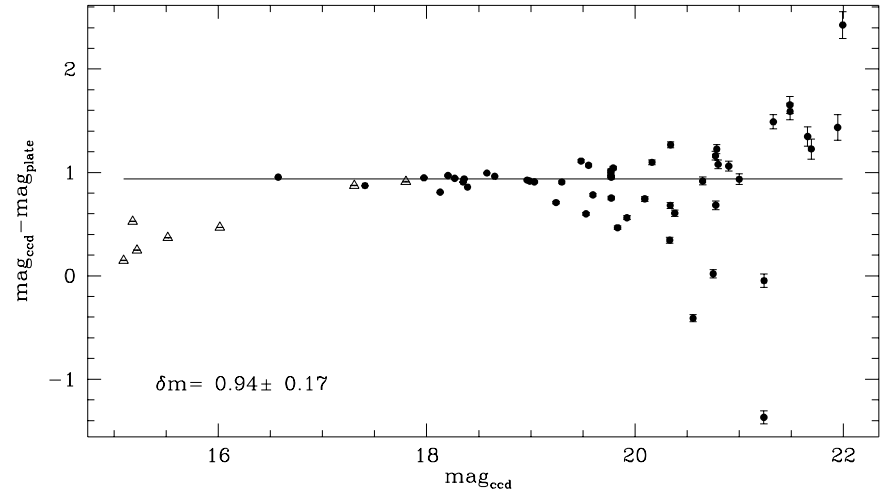

Fig. 1. Comparison between aperture instrumental magnitudes measured on the photographic plate and CCD aperture magnitudes. The continuous line represents the median difference $\delta m=\operatorname{mag}_{\text {ccd }}-$ mag $_{\text {plate }}$ for galaxies (filled dots). Stars (empty triangles) are excluded from the fit because they are often saturated on POSS II plates. This diagram has been used for calibrating the plate $F 449$

the object area is doubled. These magnitudes approximate true asymptotic magnitudes.

The plates are individually calibrated to the Gunn system (Thuan \& Gunn 1976; Wade 1979) by means of CCD frames of clusters of galaxies. We used the data set presented in Garilli et al. (1996), which has been used in GMA99 to compute the cluster LF. As they point out, their Gunn $g$ photometry does not perfectly match the standard Thuan-Gunn system (for historical reasons): $g_{\text {Garilli }}=g-0.20 \pm 0.14$. However, the error is systematic, so that we recover the true Gunn $g$ magnitude by adding this offset. We note that this is different from the general CCD calibration of DPOSS/PNSC, which is mainly based on the extensive CCD data sets obtained at Palomar for this purpose (Gal et al. 2000).

Plates are photometrically calibrated by comparing plate and CCD aperture (within 5 arcsec radius) photometry of common galaxies, and magnitudes are corrected for Galactic absorption. A typical calibration diagram is shown in Fig. 1. The adopted zero point is the median of the differences $\operatorname{mag}_{\mathrm{ccd}}-$ mag $_{\text {plate}}$, after excluding bright stars (empty triangles) that are usually saturated on photographic plates. No color term has been adopted as required by the POSS-II photometric system (Weir et al. 1995b). The mean error ${ }^{1}$ on the zero-point determination is $0.02 \mathrm{mag}$ in $g$ and $0.04 \mathrm{mag}$ in $r$ and $i$, while the typical photometric error on individual magnitudes (including Poissonian errors, residuals of density to intensity conversion, etc.) is $0.2 \mathrm{mag}$ in $g$ and 0.16 in $r$ and $i$. K-corrections were taken from Fukugita et al. (1995). Our data do not have enough resolution to distinguish between different morphological types, nor this selection can be done using galaxy colors due to the errors on individual magnitudes. Anyway the difference in k-corrections between $\mathrm{E}$ and Scd is $\leq 0.25 \mathrm{mag}$ in the $r$ and $i$ bands for the most distant

\footnotetext{
${ }^{1}$ We adopt as errors on our median zero point the semi-interquartile intervals.
} 
cluster in our sample $(\leq 0.3 \mathrm{mag}$ at our median redshift in all bands) so that we could adopt the k-correction of the dominant E-S0 population.

We estimated the photometric completeness limit of our data for each cluster and in each band independently, in order to take into account the depth variations of our catalogs from plate to plate and as a function of the projected cluster location on the plate. We adopt as our completeness limit the magnitude at which nearby field counts systematically deviate from linearity (in logarithmic units). The use of homogeneous data, reduced in one single way, both for the control field and the cluster galaxy counts, helps to partially compensate for systematic errors due to selection effects which cancel out (at least in part) in the statistical subtraction of the counts.

The studied sample is extracted from the Abell catalogue (Abell 1958; Abell et al. 1989), among those clusters with known redshift, which are imaged in a fully reduced plate triplet (i.e. $J, F$ and $N$ ) and with photometric zero points already available to us, in all three bands, at the start of this work. At that time, 39 Abell clusters satisfied the above conditions, the bottleneck being due to the low number of calibration frames and the requirement of having at least one reliable spectroscopic redshift for the cluster.

A few more clusters satisfying the above condition were also rejected from the sample on the following grounds:

Abell 154 - There are two density peaks at two different redshifts, along the line of sight, respectively at $z=0.0640$ (A154) and $z=0.0428$ (A154a).

Abell 156 - There are two discordant redshift measurements in the literature. Since there is no galaxy overdensity at the cluster position we can safely assume that it is a spurious object.

Abell 295 - Two density peaks in the cluster direction: A295 at $z=0.0424$ and A295b at $z=0.1020$.

Abell 1667 - Two density peaks in the cluster direction: A1667 at $z=0.1648$ and A1667b at $z=0.1816$.

Abell 2067 - Two density peaks in the cluster direction: A2067 at $z=0.0756$ and A2067b at $z=0.1130$.

Two more clusters, Abell 158 and Abell 259, show a double structure with two adjacent but distinct density peaks. In these cases we included only the galaxies belonging to the peaks with measured redshift, without assuming that the secondary peak lies at the same redshift as the first one. The final sample is listed in Table 1.

\section{Individual LF determination}

\subsection{Measure of the background counts and of their variance}

In order to accurately compute the cluster LF, we need to statistically subtract the background from galaxy counts in the cluster direction. This step requires particular attention to three potential sources of errors:
Table 1. The cluster sample

\begin{tabular}{ccccc}
\hline Cluster & Redshift & Plate & Richness class & B-M type \\
\hline A1 & 0.1249 & 607 & 1 & III \\
A16 & 0.0838 & 752 & 2 & III \\
A28 & 0.1845 & 680 & 2 & III \\
A41 & 0.2750 & 752 & 3 & II-III \\
A44 & 0.0599 & 680 & 1 & II \\
A104 & 0.0822 & 474 & 1 & II-III \\
A115 & 0.1971 & 474 & 3 & III \\
A125 & 0.188 & 610 & 1 & III \\
A150 & 0.0596 & 610 & 1 & I-II \\
A152 & 0.0581 & 610 & 0 & $\ldots$ \\
A158 & 0.0645 & 610 & 0 & $\ldots$ \\
A180 & 0.1350 & 755 & 0 & I \\
A192 & 0.1215 & 755 & 2 & I \\
A202 & 0.1500 & 755 & 2 & II-III \\
A267 & 0.2300 & 829 & 0 & $\ldots$ \\
A279 & 0.0797 & 829 & 1 & I-II \\
A286 & 0.1603 & 829 & 2 & II \\
A293 & 0.1650 & 757 & 2 & II \\
A294 & 0.0783 & 757 & 1 & I-II \\
A1632 & 0.1962 & 443 & 2 & II-III \\
A1661 & 0.1671 & 443 & 2 & III \\
A1677 & 0.1845 & 443 & 2 & III \\
A1679 & 0.1699 & 443 & 2 & III \\
A1809 & 0.0788 & 793 & 1 & II \\
A1835 & 0.2523 & 793 & 0 & $\ldots$ \\
A2049 & 0.1170 & 449 & 1 & III \\
A2059 & 0.1305 & 449 & 1 & III \\
A2061 & 0.0782 & 449 & 1 & III \\
A2062 & 0.1122 & 449 & 1 & III \\
A2065 & 0.0721 & 449 & 2 & III \\
A2069 & 0.116 & 449 & 2 & II-III \\
A2073 & 0.1717 & 449 & 1 & III \\
A2083 & 0.1143 & 449 & 1 & III \\
A2089 & 0.0743 & 449 & 1 & II \\
A2092 & 0.066 & 449 & 1 & II-III \\
A2177 & 0.1610 & 517 & 0 & $\ldots$ \\
A2178 & 0.0928 & 517 & 1 & II \\
A2223 & 0.1027 & 517 & 0 & III \\
A2703 & 0.1144 & 607 & 0 & $\ldots$ \\
\hline & & & &
\end{tabular}

1) Catalogs should be complete and clean from spurious detections: one single entry in the catalog should correspond to each object in the sky and vice versa. Unfortunately, single-filter SKICAT catalogs contain numerous faint spurious objects, mainly around bright sources. This is due to the fact that SKICAT was intentionally tailored to detect even the faintest objects 


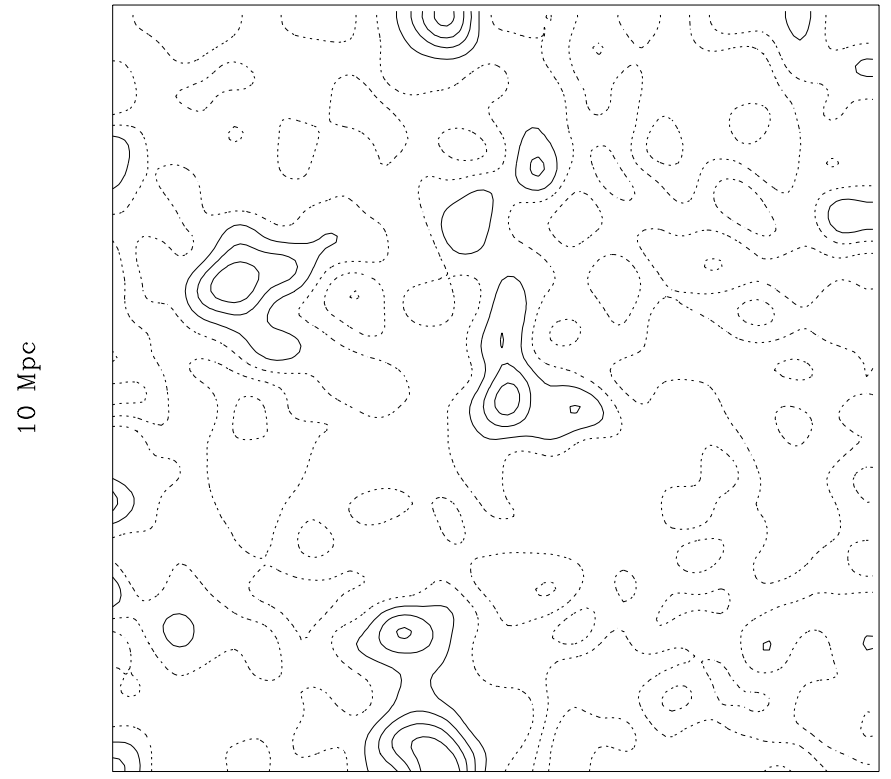

$10 \mathrm{Mpc}$

Fig. 2. Density map of a $10 \times 10 \mathrm{Mpc}$ region around Abell 152. The continuous and dotted lines represent density levels respectively above and under the $1.5 \sigma$ level used to detect the cluster area (Sect. 3.2). Levels are spaced $1 \sigma$ apart

and therefore pays the price of detecting false objects in the halo of saturated or extended sources.

All objects in a $10 \times 10 \mathrm{Mpc}$ region centered on the cluster center (as estimated by Abell 1958), are thus extracted from the three calibrated catalogs (in $g, r$ and i) and positionally matched using their right ascension and declination. False detections are removed in the matching step, due to the low probability that two (or even three) false detections occur in the same sky position in different filters. The maximum allowed distance for the matching was fixed at 7 arcsec in order to take into account the positional uncertainty (2.1 arcsec at $95 \%$ confidence level, Deutsch 1999, for a single filter) while minimizing the number of erroneous matches (the average distances between galaxies in the cluster regions is 5 times our matching distance). As a further precaution, we excluded a circular region (with an area five times larger than the isophotal area) around each potential troublemaker (bright stars and very extended objects). The coordinates of the removed areas were stored to allow for later corrections. The total area removed constitutes, at most, $3 \%$ of the overall area. The final catalogue was then used to produce a galaxy surface density map which (in order to enhance structures at the cluster scale, Fig. 2) was convolved with a Gaussian function having $\sigma$ of $250 \mathrm{kpc}$ - i.e. the size of a typical core radius - in the cluster rest frame;

2) Counts in the cluster and control field directions should be accurately photometrically calibrated without relative systematic errors. We achieve this requirement by using two different portions of the same image;

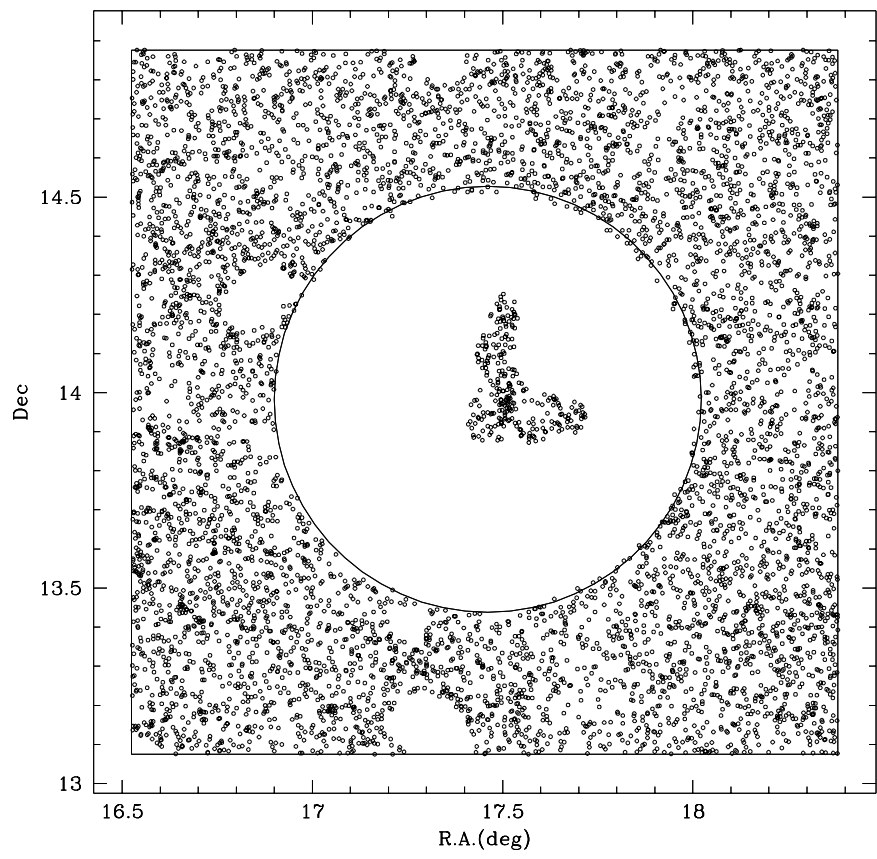

Fig. 3. The Abell 152 cluster+background field. Dots within the inner circle represent galaxies included in the $1.5 \sigma$ region, while those outside the circle are background galaxies. The empty regions in the background area represent the removed overdensity regions (Sect. 3.1). The region is the same as shown in Fig. 2

3) Field counts should be computed in a region far enough from the clusters to not be contaminated by cluster galaxies, but near enough to take into account nonuniformities of the field on the scale of the angular size of the cluster. For this purpose we take advantage of our wide field capabilities by dividing the $10 \times 10 \mathrm{Mpc}$ region in two parts: an inner circle of $3 \mathrm{Mpc}$ radius, i.e. 1 Abell radius $\left(R_{\mathrm{A}}=1.5 h^{-1} \mathrm{Mpc}\right)$, used to search for the cluster overdensity, and an external region, beyond the $3 \mathrm{Mpc}$ circle and within the $10 \mathrm{Mpc}$ square, where we measure the field counts (Fig. 3). This radius is large enough that the contamination of cluster galaxies in the control field line of sight is minimal and the $\mathrm{S} / \mathrm{N}$ of the LF is not significantly reduced (if at all). In fact, the cluster overdensity is undetected at these radii for the large majority of the clusters.

However, a control field too near the cluster, while diminishing the $\mathrm{S} / \mathrm{N}$ of the $\mathrm{LF}$ determination, does not alter the shape of the LF. In fact:

$$
\begin{aligned}
N_{\mathrm{cl}}(m) & =N_{\mathrm{cl}+\mathrm{bkg}}(m)-N_{\mathrm{bkg}}(m) \\
& =\left(N_{\mathrm{cl}}(m)+N_{\mathrm{bkg}}(m)\right)-N_{\mathrm{bkg}}(m)
\end{aligned}
$$

where $N_{\mathrm{cl}}(m)$ is the number of cluster galaxies at a certain magnitude $m$ and $N_{\mathrm{bkg}}(m)$ is the number of background galaxies at the same magnitude. A too near control field is contaminated by the cluster galaxies, i.e.

$N_{\mathrm{bkg}}^{\mathrm{near}}(m)=N_{\mathrm{bkg}}(m)+\gamma \cdot N_{\mathrm{cl}}(m)$ 

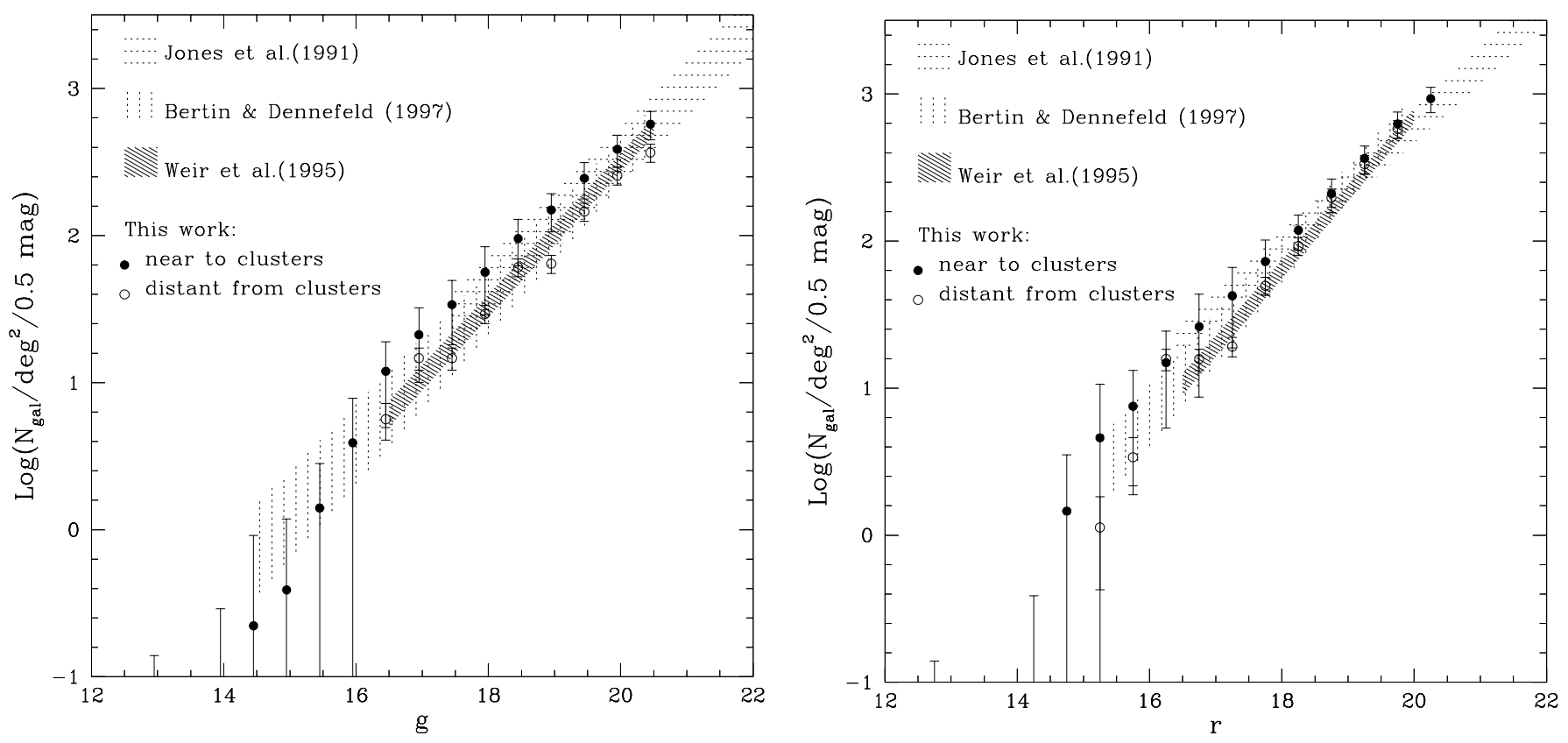

Fig. 4. Mean background counts in regions near and far from clusters, compared to literature, in the $g$ and $r$ band (see discussion in text). Literature counts fall within the shaded regions

where $\gamma$ is the ratio between the cluster galaxy density in the cluster region and in the control field direction. Therefore using $N_{\mathrm{bkg}}^{\mathrm{near}}(m)$ instead of $N_{\mathrm{bkg}}(m)$, one obtains from Eq. (1):

$$
\begin{aligned}
N_{\mathrm{cl}}^{\text {near }}(m) & =N_{\mathrm{cl}+\mathrm{bkg}}(m)-\left(N_{\mathrm{bkg}}(m)+\gamma \cdot N_{\mathrm{cl}}(m)\right) \\
& =N_{\mathrm{cl}}(m) \cdot(1-\gamma)
\end{aligned}
$$

i.e., by using a too near control field, the number of cluster galaxies (and thus the LF if the applied k-correction does not depend on galaxy type, as in our case) is simply diminished by a multiplicative factor and its shape is not affected. To be more precise, one should note that we are supposing that the LF shape does not depend on the cluster location or that the contamination is very small, so that even large differences in the LF have null impact.

Field galaxy counts are measured in the external region once the removed areas are taken into account. Furthermore, in order not to bias the average background due to the existence of other groups and clusters, we remove every density peak above $2 \sigma$ from the mean field density (see Fig. 3). The average, which we call the "local field", is the adopted estimate of the background counts in the cluster direction.

The "local" field, computed all around the cluster, is a better measure of the contribution of background galaxies to counts in the cluster direction than the usual "average" field (measured on a single spot and/or far from the considered cluster), since it allows us to correct for the presence of possible underlying large-scale structures both at the cluster distance and in front of or behind it.

Figure 4 shows that our galaxy counts are consistent with previous determinations, and in particular with those by Weir et al. (1995b), who also made use of DPOSS plates. Nevertheless, counts near clusters (filled circles), but not too near to be affected by them, tend to be systematically higher than the average and in particular of those extracted in a reference region particularly devoid of structures (empty circles), even if differences are within the errors. This difference can be as high as $80 \%$ of the mean value. The higher value can be explained by the fact that we are sampling the superclusters surrounding the studied clusters, whose contribution can be missed when measuring background in smaller and random fields, as often done in the literature.

Once the background to be subtracted from cluster counts has been determined, we need a robust evaluation of the error involved in the subtraction process. There are three sources of errors: Poissonian errors for galaxies belonging to the cluster, plus Poissonian and non-Poissonian fluctuations of the background counts.

Poissonian fluctuations in the number of cluster members are significant only at magnitudes where the control field counts have close to zero galaxies per bin. Poissonian fluctuations of the background in the control field direction are small because of the large area used to determine the local field (at least 20 times larger). Therefore, the dominant term in the error budget is due to the nonPoissonian fluctuations of background counts along the cluster line of sight. The wide coverage of the DPOSS fields allow us to easily and directly measure the variance of galaxy counts, and thus the field fluctuations (Poissonian and non-Poissonian) on the angular scale of each individual cluster in adjacent directions, instead of relying on model estimates (e.g. Huang et al. 1997). It should be noted that, until a few years ago, non-Poissonian fluctuations were often completely ignored, thus underestimating the errors on the LF. 


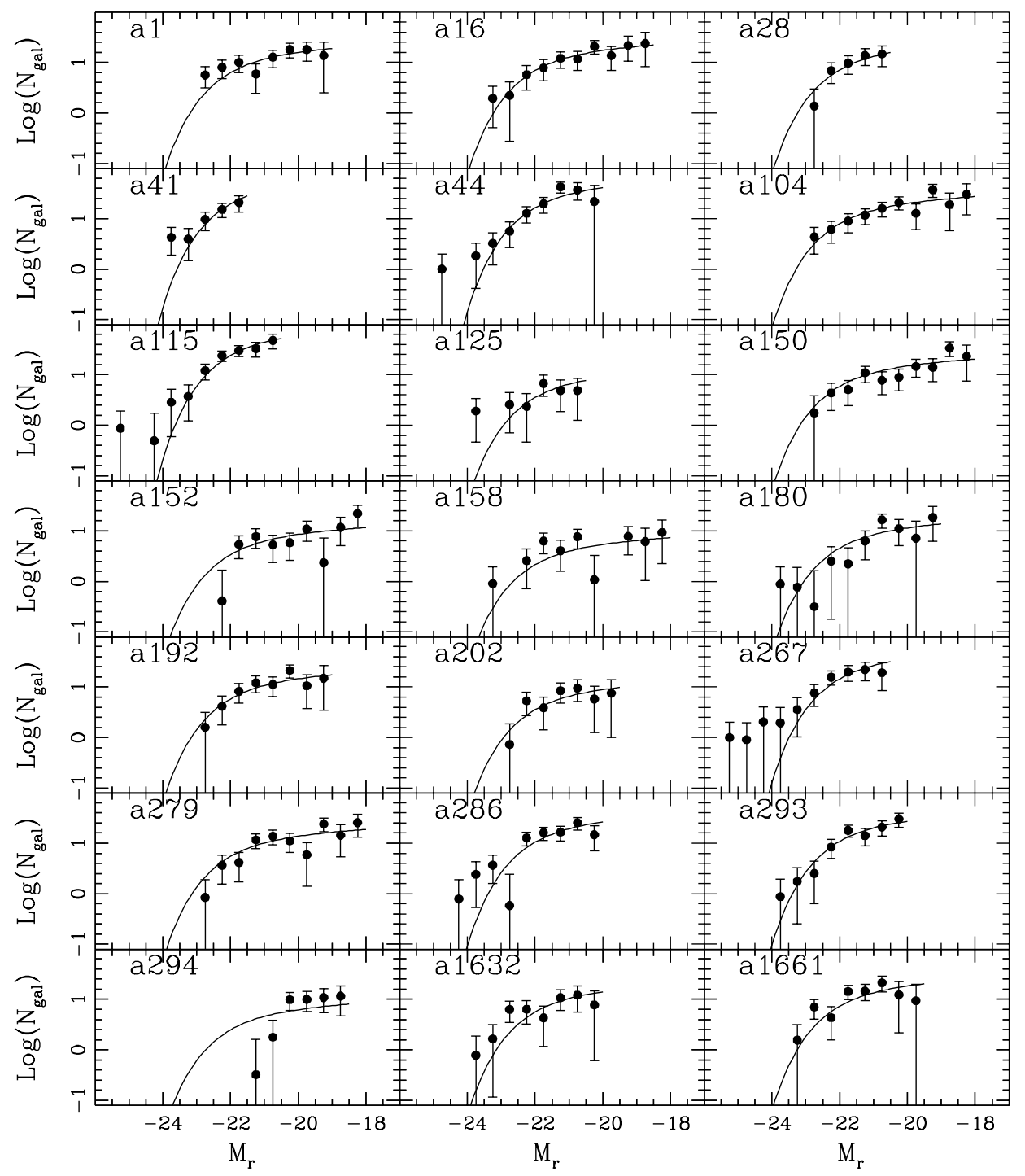

Fig. 5. a) The background-corrected galaxy counts for the first 21 cluster of our sample in the $r$ band. The best-fit Schechter function of the cumulative LF (par. 4), normalized to the total counts in each cluster, is shown as a continuous line

Adami et al. (1998) questioned this statistical method of computing the LF (a method that dates back at least to Zwicky 1957), and checked the validity of a statistical field subtraction by means of a redshift survey in the case of one single cluster, finding a discrepancy between the counts of Bernstein et al. (1995) and those inferred from spectroscopic measurements. They argued that the statistical method can be affected by potential errors. Nevertheless their narrow field of view $\left(7.5^{\prime} \times 7.5^{\prime}, \sim 280 \times 280 \mathrm{kpc}^{2}\right.$ at cluster distance) and the small number of galaxies (49) in their sample, does not allow to draw any significant conclusion from this test (the observed discrepancy is statistically significant only at the $1 \sigma$ level).

Furthermore, the fact that they sampled the core of a rich cluster where other effects - as they notice - such as tidal disruption might be dominant, and the possibility that the Bernstein et al. field counts could be underestimated (the use of random fields to measure background does not take into account the presence of underlying large-scale structure), contribute to bringing the discrepancy well below $1 \sigma$.

\subsection{The individual $L F$}

Due to the low (1 arcmin) astrometric accuracy of the Abell (1958) cluster centers, we first search for the central $1.5 \sigma$ density peak in the inner $3 \mathrm{Mpc}$ circle (much larger than 1 arcmin at all considered redshifts) centered on the approximate cluster position as determined by Abell (Fig. 3). We then derive the cluster LF by subtracting, from the galaxy counts measured in this region, the local field counts, rescaled to the effective ${ }^{2}$ cluster area.

\footnotetext{
${ }^{2}$ I.e. the area corrected for the regions removed around the troublemakers.
} 


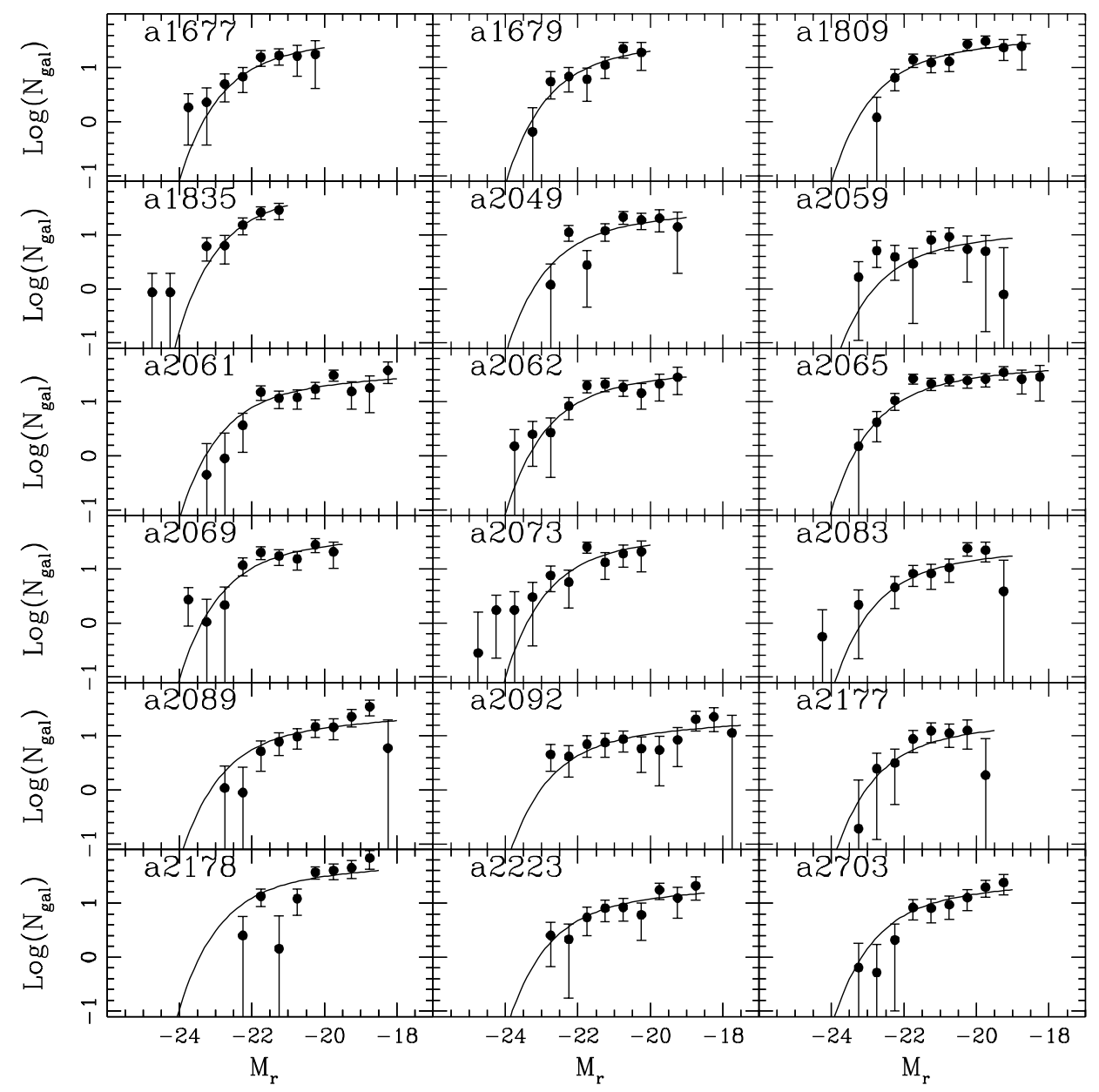

Fig. 5. b) As in Fig. 5a for the remaining 18 clusters

This approach allows us to take into account the cluster morphology without having to adopt a fixed cluster radius, and thus to apply the local field correction to the region where the signal (due to the cluster) to noise (due to field and cluster fluctuations) ratio is higher, in order to minimize statistical uncertainties.

The LF for individual clusters in the $r$ band are shown in Figs. 5a,b, together with the best-fit Schechter function of the composite LF (Sect. 4). Because we already used the whole cluster for computing the LF, individual LFs cannot be improved further, except by performing expensive redshift surveys.

\section{Results}

Most of our clusters have too few galaxies to accurately determine the shape of the LF. Instead, we can combine all individual LFs to construct the composite LF of the whole sample. In doing so, vagaries of individual LFs are washed out and only the underlying possibly universal LF is enhanced. We adopt the method used by GMA99, consisting of a modified version of the formula introduced by Colless (=C89, 1989). In practice, the composite LF is obtained by weighting each cluster against the relative number of galaxies in a magnitude range that takes into account the variations in the completeness limit of our data.

Ostriker \& Hausman (1977) have shown that giant galaxies in clusters may be the result of peculiar accretion processes. For this reason we took care to remove from each cluster the Brightest Cluster Member (BCM).

The final composite LF is shown in Fig. 6 for the $g, r$ and $i$ bands.

The fit of the composite luminosity functions to a Schechter (1976) function ${ }^{3}$

$$
N(M)=\Phi 10^{0.4\left(M^{*}-M\right)(\alpha+1)} \exp \left(10^{0.4\left(M^{*}-M\right)}\right)
$$

gives the values listed in Table 2 , where $M^{*}$ is the characteristic knee magnitude and $\alpha$ is the slope of the LF at

\footnotetext{
3 The function has been convolved with a boxcar filter in order to take into account the finite amplitude of the magnitude bins.
} 

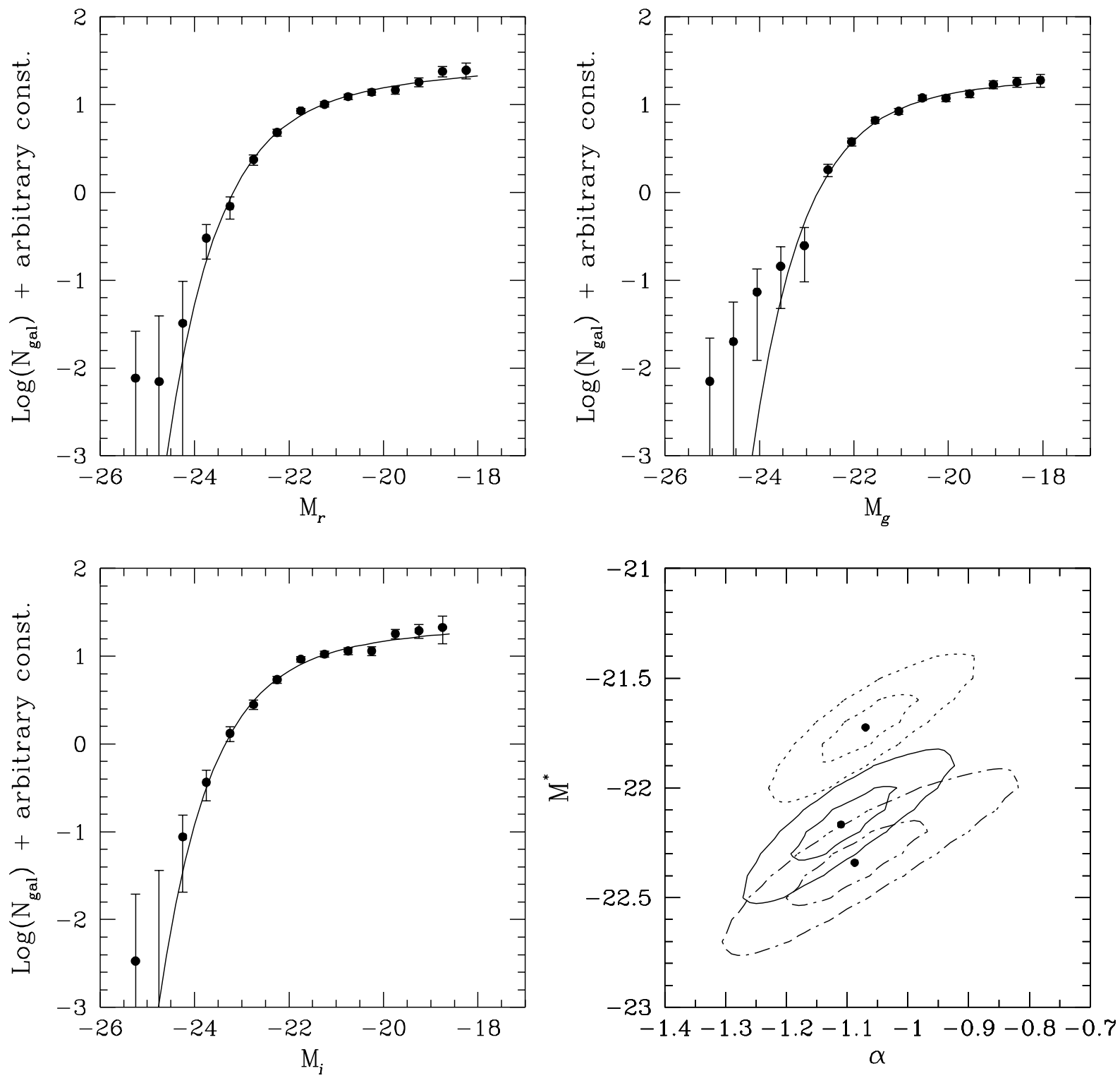

Fig. 6. The composite LF in the $g, r$ and $i$ bands obtained excluding the brightest member of each cluster (filled dots). The best fit Schechter functions are represented by continuous lines, with the $68 \%$ and $99 \%$ confidence levels of the best fit parameters in the bottom right panel ( $g$ : dotted line; $r$ : continuous line; $i$ : dashed-dotted line)

Table 2. LF best fit Schechter parameters. The given errors are referred to $1 \sigma$ confidence levels

\begin{tabular}{cccc}
\hline Band & $\alpha$ & $M^{*}$ & $\chi^{2} /$ d.o.f. \\
\hline$g$ & $-1.07_{-0.07}^{+0.09}$ & $-21.72_{-0.17}^{+0.13}$ & $9.4 / 13$ \\
$r$ & $-1.11_{-0.07}^{+0.09}$ & $-22.17 \pm 0.16$ & $10.2 / 13$ \\
$i$ & $-1.09_{-0.11}^{+0.12}$ & $-22.35 \pm 0.20$ & $11.4 / 12$ \\
\hline
\end{tabular}

faint magnitudes. Figure 6 shows the three best-fit functions together with the $68 \%$ and $90 \%$ confidence levels. The LFs turn out to be quite well described by a Schechter function in our magnitude range (see $\chi^{2}$ in Table 2 ).
The faint end of the composite LF $\left(g:-1.07_{-0.07}^{+0.09}, r\right.$ : $\left.-1.11_{-0.07}^{+0.09}, i:-1.09_{-0.11}^{+0.12}\right)$ is, in all bands, shallower than the traditional value, $\alpha=-1.25$ (cf. Schechter 1976), but still compatible within the $99 \%$ level in the $r$ and $i$ bands. The best fit values of $\alpha$ in the three bands are almost identical, while $M^{*}$ increases from the blue to the red band as it is expected from the color of the dominant population in clusters (taken, for example, from Fukugita et al. 1995).

In order to test if our background is measured too near the cluster, we re-computed the LF by adopting the $g$ and $r$ field counts derived by Weir et al. (1995b), from the same photographic material and using the same software. We also adopt our direct measure of the background 
Table 3. The best-fit Schechter parameters for the meanbackground corrected LF

\begin{tabular}{cccc}
\hline Band & $\alpha$ & $M^{*}$ & $\chi^{2} /$ d.o.f. \\
\hline$g$ & $-1.11 \pm 0.07$ & $-21.87 \pm 0.13$ & $9.7 / 13$ \\
$r$ & $-1.12 \pm 0.06$ & $-22.20 \pm 0.13$ & $11.2 / 13$ \\
\hline
\end{tabular}

fluctuations, because these are not provided in Weir et al. The newly found best fit parameters differ by less than $1 \sigma$ from those previously determined, thus suggesting that cluster members that are more than $3 \mathrm{Mpc}$ away from the clusters (and that therefore fall in our control field direction) have null impact on the composite LF. A definitive assessment of the effects of this assumption on the outer $\mathrm{LF}$, which is much more sensitive to a small error on the background correction, calls, however, for a larger sample of clusters.

We stress that for the time being we prefer to use the local background for an aesthetic reason: the use of the far field implicitly assumes that all galaxy overdensities near the cluster belong to the cluster, including superclusters and filaments. From a technical point of view, the problem is similar to the well understood problem of performing accurate photometry of non isolated objects: when an object in embedded in (or simply superposed to) a much larger one as it happens, for instance, in the case of HII regions or globular clusters on a galaxy or in that of a small galaxy projected on the halo of a larger one. It makes no sense to measure the background very far from the source of interest, since such a procedure ignores the non negligible background contributor. By using a "far distant" background field, we would produce perfectly empty regions at the location of clusters in superclusters, for HII regions in galaxies, and at every locations in the Universe where there are superposed structures of different sizes.

\subsection{Comparison with previous determinations}

Our composite $g$ and $r$ LFs can be easily compared with those obtained from photographic material by C89 in the $B_{\mathrm{J}}$ band and by Lugger (=L86, 1986) in the $R$ band, as shown in Fig. 7. Conversions between their photometric systems and our own has been performed using the color conversions given in the original papers and those by Fukugita et al. (1995). We found that the characteristic magnitudes agree very well (within $1 \sigma$ ) while the faint end slopes are compatible within $2 \sigma\left(\alpha_{\mathrm{C} 89}=-1.21\right.$ and $\left.\alpha_{\mathrm{L} 86}=-1.24\right)$. At bright magnitudes our LF matches the Lugger one well, but not the Colless one, which includes the BCMs in the LF. Anyway, our LF extends more than one magnitude further both at the bright and faint end: the bright end, which includes rare objects, is better sampled due to the large area coverage of our survey, whereas fainter magnitudes are reached due to our deeper magnitude limit.

Evidence in favor of a flat LF has been presented by many authors (cf. Gaidos 1997 for 20 Abell clusters and
GMA99 for 65 clusters). A comparison with GMA99 is of particular interest since, in addition to adopting our same photometric system, they use a completely different method for removing possible interlopers. GMA99 exploit the fact that the observed colors of the galaxies change with redshift due to the $\mathrm{k}$-correction, which moves the background objects in a locus of the color-color plane different from that occupied by the cluster galaxies. We compare our $r$ band LF with GMA99 in Fig. 8. The agreement is impressive considering not only that the background correction is made using different approaches, but also the different total magnitude corrections (FOCAS "total" in this work, aperture magnitude corrected to total in GMA99). Moreover, our sample is independent from theirs, except for a few clusters wich are anyway sampled in different regions due to the different field of view.

We find that both the slope and the characteristic magnitude of their best-fit function are in good agreement with ours and are compatible within the errors (within $2 \sigma$ ). This agreement tends to confirm that our choice of using a local background determination instead of the "average" one leads to a good estimate of the number of interlopers contaminating cluster galaxy counts.

In the comparison with GMA99, a few more facts are worth mentioning:

- we reach a similar determination of the cluster LF at lower telescope time price: using just a few $(\sim 10)$ plates taken for a very general purpose (a sky survey) with a Schmidt telescope, we achieve the same performances as in a multi-year CCD campaign on a $2 \mathrm{~m}$ class telescope;

- our LF extend to brighter magnitudes, thus sampling the LF at the location of rare objects, a possibility allowed only by large area surveys.

Even though CCD data are usually deeper and have higher photometric accuracy than ours, they are also limited to small regions of the clusters and usually cover different cluster portions at different redshifts. Our data, instead, cover the whole cluster area independently of the redshift, but are selected in apparent magnitude. This means that the fainter magnitude bins of the composite LF, are populated mainly by the galaxies in the nearer clusters. In absolute-magnitude selected surveys instead, the faintest bins usually include preferentially distant galaxies, due to the large area covered at high redshift with a fixed field of view. This is not true for GMA99, where the redshift distribution was quite uniform since the authors tried to observe nearby clusters with a large field of view and distant clusters are slightly less deeply probed than the near ones. However, the total number of objects in our sample is approximately 1.5 times the number of objects in the GMA99 sample.

Our result disagrees with the steep $(-1.6<\alpha<-1.4)$ LF found by Valotto et al. (1997). Their work is based on photographic data taken from the APM cluster survey and they adopt, as we do, "local" background counts measured in annuli surrounding each cluster. Nevertheless, 

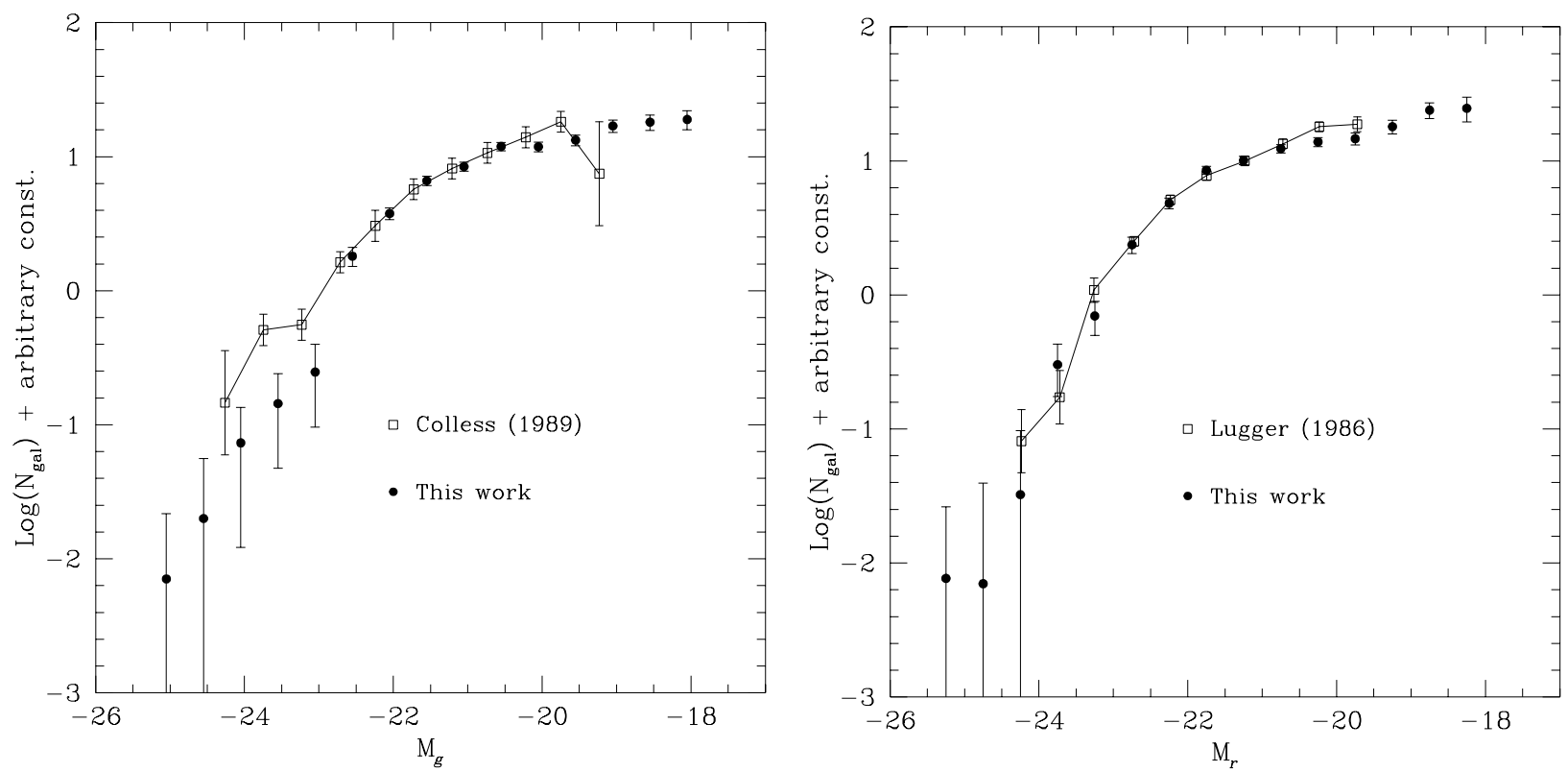

Fig. 7. Comparison of our composite LF with those of Colless (1989) in the $g$ band and Lugger (1986) in the $r$ band, both based on photographic data. Literature LF have been vertically shifted to match our LF

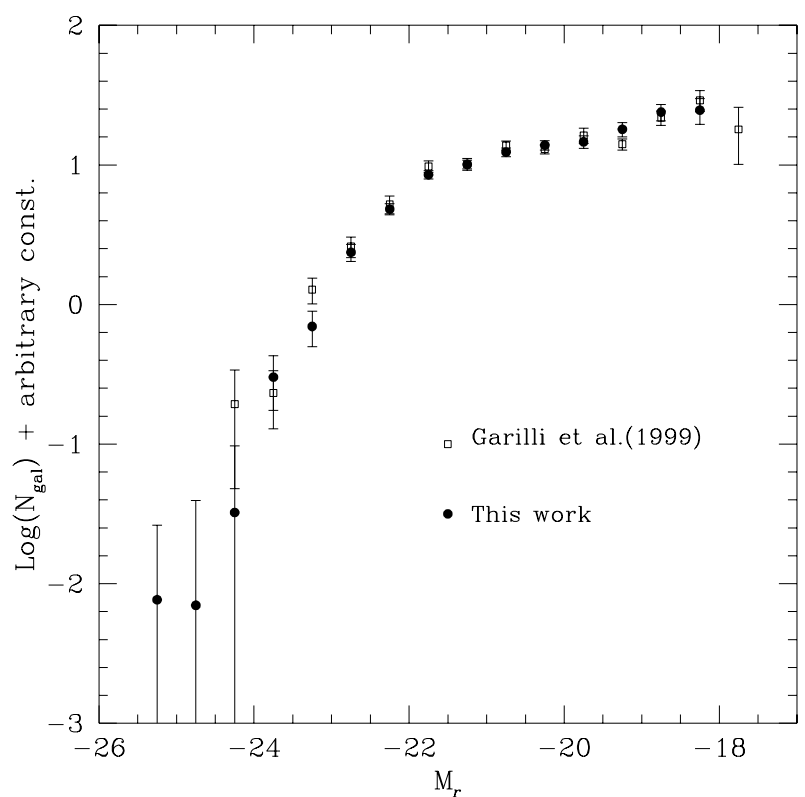

Fig. 8. Comparison between our composite LF and the Garilli et al. (1999) LF obtained from CCD data and adopting a different method to remove interlopers (see discussion in text)

their completeness limit is 1.5 magnitudes shallower than ours, so that they are sampling the brighter portion of the LF, and therefore the slope is subject to large errors.

At first glance, our claim that a Schechter function is a good fit to our data $\left(\chi^{2} \leq 1\right)$ seems in contradiction with various claims of a non-universal LF produced by the various morphological composition of clusters and by the non-homology of the LFs of the morphological types (e.g. Sandage et al. 1985; Jerjen \& Tammann 1997; Andreon 1998) or because of the variable dwarf content of clusters (Secker \& Harris 1996; Trentham 1997, 1998).

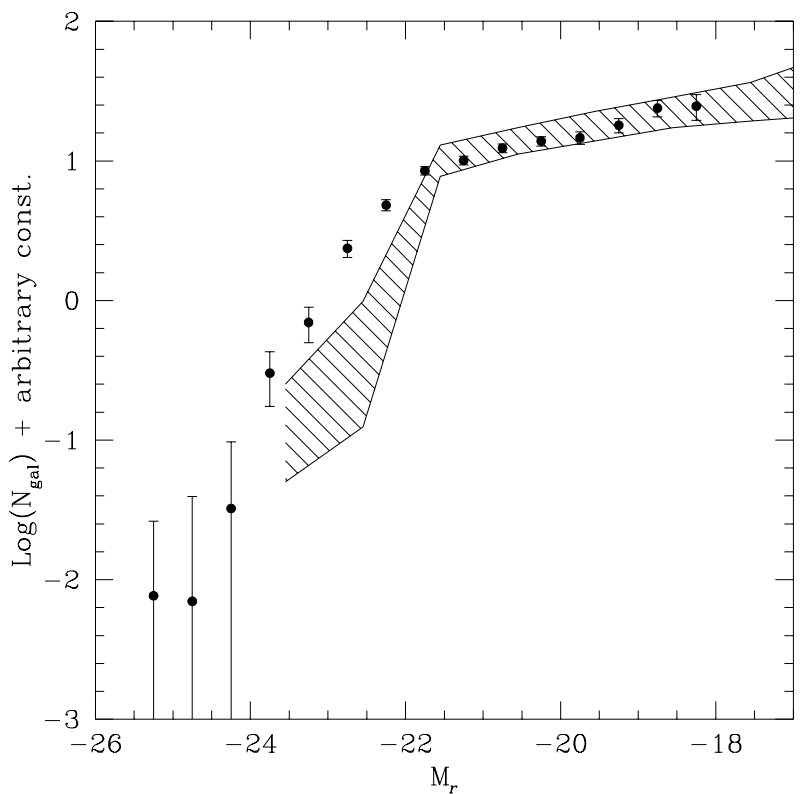

Fig. 9. Comparison between our composite LF (filled dots) and the Trentham (1997) LF (shaded region) based on CCD data

Trentham (1997), for instance, showed that the cluster $\mathrm{LF}$ rises steeply at faint magnitudes $\left(M_{\mathrm{g}}>-18\right)$ and thus a simple Schechter function cannot properly describe the whole distribution. Nevertheless, as shown in Fig. 9, for magnitudes between -22 and -17 the LF is quite flat and in good agreement with our data. In fact, in our magnitude range the contribution of dwarf galaxies is visible only in the faintest bins, as suggested by the fact that in Fig. 6 the last points lie systematically above the bestfit function. This trend (a flattening of the distribution around $M=-21$ and a steepening over $M=-19.5$ ) is also confirmed by the comparison with GMA99, whose LF 
Table 4. Results of $\chi^{2}$ tests for different subsamples

\begin{tabular}{|c|c|c|c|c|c|c|}
\hline & \multicolumn{6}{|c|}{ Band } \\
\hline & \multicolumn{2}{|c|}{$g$} & \multicolumn{2}{|c|}{$r$} & \multicolumn{2}{|c|}{$i$} \\
\hline & $\chi^{2} /$ d.o.f. & Prob. $>\chi^{2}$ & $\chi^{2} /$ d.o.f. & Prob. $>\chi^{2}$ & $\chi^{2} /$ d.o.f. & Prob. $>\chi^{2}$ \\
\hline$R>1$ vs. $R \leq 1$ & $22.98 / 16$ & 0.11 & $6.58 / 15$ & 0.97 & $11.38 / 14$ & 0.66 \\
\hline BM I+I-II vs. II+II-III & $21.91 / 15$ & 0.11 & $13.18 / 12$ & 0.36 & $11.43 / 11$ & 0.41 \\
\hline BM I+I-II vs. III & $22.66 / 14$ & 0.07 & $20.81 / 12$ & 0.05 & $13.29 / 11$ & 0.27 \\
\hline BM II+II-III vs. III & $9.45 / 15$ & 0.85 & $9.37 / 14$ & 0.80 & $4.18 / 12$ & 0.98 \\
\hline Compact vs. Elongated & $20.47 / 14$ & 0.12 & $11.78 / 14$ & 0.62 & $14.59 / 13$ & 0.33 \\
\hline Compact vs. Multiple & $17.74 / 17$ & 0.41 & $6.92 / 15$ & 0.96 & $17.97 / 14$ & 0.21 \\
\hline Elongated vs. Multiple & $20.03 / 14$ & 0.12 & $11.49 / 14$ & 0.65 & $10.13 / 12$ & 0.60 \\
\hline
\end{tabular}

shows a similar behavior. At bright magnitudes, instead, the act of averaging over the cluster region can mask the environmental effects.

We must note that while in Fig. 9 the two LFs differ substantially at the bright end, our data are in very good agreement with L86 and GMA99, thus suggesting that Trentham is underestimating the contribution of bright galaxies to the LF. This can be due to various reasons, including the small area and the specific portions of the clusters sampled, or the different morphological composition of his clusters. Moreover, due to our larger number of clusters, we can sample the LF at twice the resolution in magnitude.

\subsection{Dependence on the cluster physical parameters}

We compared the LFs obtained dividing our sample into rich (Abell class $R>1$ ) and poor $(R \leq 1)$ clusters. Table 4 shows that the LFs of these two classes are consistent within the errors. GMA99 found instead that the slope of the LF computed in the central regions of the clusters depends on the cluster central density, while they found mild differences, statistically significant, between rich and poor clusters. Our result differs from the GMA99 finding that the giant to dwarf ratio is higher in rich clusters than in poor ones, but only in the statistical significance: we find that poor clusters have a faint-end slope steeper than rich clusters $(\overline{\Delta \alpha}=0.16)$ by a quantity that is compatible within $1 \sigma$ to those derived by GMA99 in their poor-rich comparison. The dependence of the slope on richness is more evident in the $g$ band, as shown in Fig. 10.

We also explored the influence of the cluster dynamical state, as indicated by the Bautz-Morgan type (Bautz \& Morgan 1970), on the LF. We divided the sample in three subsamples: BM I + BM I-II, BM II + BM II-III and BM III in order to have a similar number of clusters in each group. We find that early and late BM types have LFs which are compatible within 95\%, in agreement with GMA99 and Lugger (1986).

Moreover, we divided our sample into 3 morphological classes based on visual inspection of density profiles (cf. Sect. 4.1). We classified clusters into "compact",

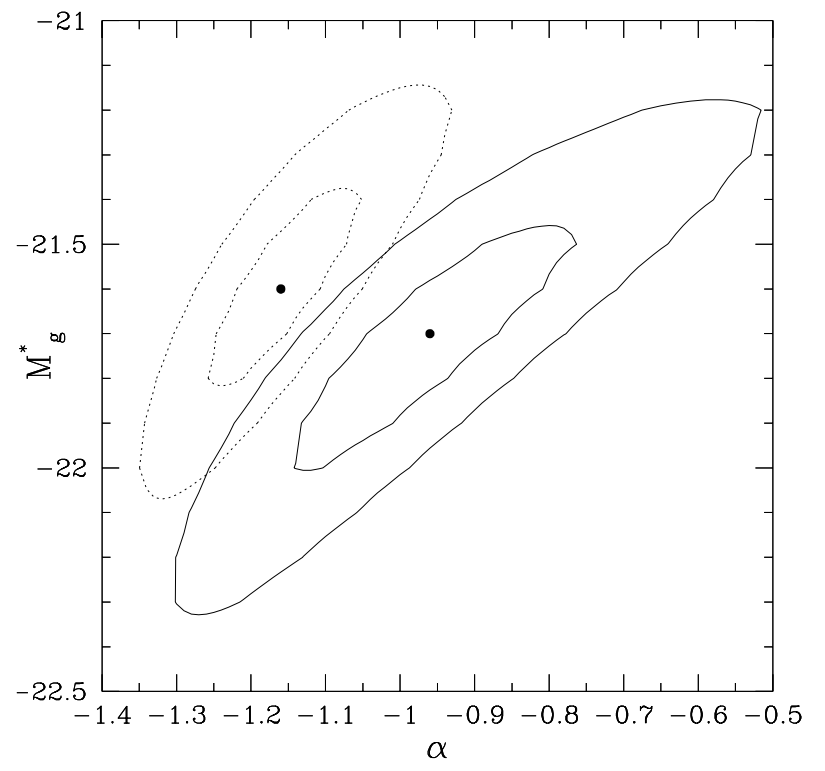

Fig. 10. The $68 \%$ and $99 \%$ confidence levels relative to the fit of the rich $(R>1$, continuous line) and poor $(R \leq 1$, dotted line) subsamples in the $g$ band

showing a single strong density peak within the $1.5 \sigma$ isodensity contour above background; "elongated" if the cluster is irregularly spread across the field with a weak density peak, and "multiple" if it shows multiple peaks. Again, we find no significant differences between the LFs of these classes of clusters.

In interpreting this result we note that when our sample is divided in subsamples the number of objects may not be large enough for a $\chi^{2}$ test to reveal differences in the distribution, as in the case of the poor-rich comparison, so that a conclusive statement calls for a larger sample.

\subsection{Comparison with the field $L F$}

As already shown in GMA99, we find that the cluster LF is compatible with the field LF. This result does not rule out environmental influence on galaxy formation and/or evolution, but rather indicates that either evidence for such effects must be investigated at fainter magnitudes than 


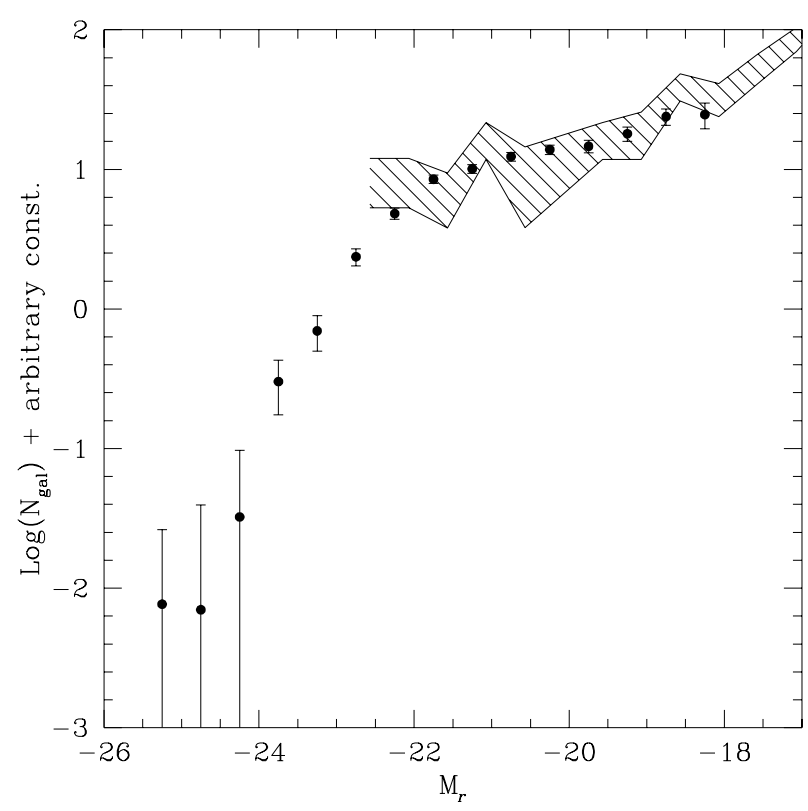

Fig. 11. Comparison between our composite LF (filled dots) and the LF obtained by Andreon \& Cuillandre (2000), (shaded region)

those reached by DPOSS data, or that the effect is smaller than what the data allows us to detect.

\subsection{Compact galaxies misclassified?}

It could be argued that our (and also most literature) LF are flatter than they should be since compact galaxies are misclassified as stars. For the most distant cluster even normal galaxies are badly classified due to the low angular resolution of the available images and/or to errors in the star/galaxy classifiers (see, for example Drinkwater et al. 1999). In our case, the comparison with the LF of the Coma cluster obtained by Andreon \& Cuillandre (2000) settles this issue, because their determination is not affected from this problem since it does not use any star/galaxy classification. Figure 11 shows the good agreement between the two LFs and confirms that we are not missing any large population of compact galaxies.

\section{Conclusions}

We computed the composite LF of 39 clusters of galaxies at $0.08<z<0.3$ in three filters from the DPOSS plates, using the well known fact that clusters are galaxy overdensities with respect to the field. Our LF agrees with previous determinations of the cluster LF, obtained using specifically tailored observations, while we use sky survey plate data. The LFs are well described by a Schechter function, with a shallow slope $\alpha \sim-1.1$ with minor variations from blue to red filters and $M^{*} \sim-22.4,-22.2,-21.7$ $\left(H_{0}=50 \mathrm{~km} \mathrm{~s}^{-1} \mathrm{Mpc}^{-1}\right)$ in $g, r$ and $i$ filters, respectively. The LFs are computed without the assumption of an average background along the cluster line of sight, and use actual measurement of the background fluctuation instead of relying on the formalism and hypothesis presented in Huang et al. (1996) or, as in older works, assuming an "average" error. The existence of compact/misclassified galaxies have no impact on our LF determination: they are a minority population or a magnitude independent fraction of the number other galaxies.

The similarity of composite LFs by GMA99, measured from CCD photometry of the cluster central regions, suggests minor differences between the LF in the cluster outskirts and in the central one, or a minor contribution of galaxies in the cluster outskirts to the global LF.

When our cluster sample is grouped in classes of richness, dynamical and morphological type, we find no significant differences among the classes. However, our cluster sample may be not large enough for detecting the differences found in other studies, or the differences may be intrinsically too small to be detected in a sample, like ours, which is large but not huge (and the latter sample still does not exist).

Our results on the cluster LFs are not completely new: other authors found similar results, and for this reason we avoid repeating the cosmological implication of our results. However, we wish to stress that: we have a better control of the errors, due to the nearby control field and the direct measure of the field variance; we identify in the literature a few discrepant LFs in certain magnitude ranges; we show that the statistical subtraction of the background is sound, since we found the same LF shape found by Garilli et al., who removed interlopers by adopting an independent method; we obtain these results by using all-purpose photographic plates, instead of a multiyear CCD campaign.

We are currently increasing the present sample by an order of magnitude in order to explore with greater statistical significance the dependence of the cluster LF on the physical parameters.

Acknowledgements. Bianca Garilli, Dario Bottini and Dario Maccagni are warmly thanked for providing us with the electronic access to the data shown in Fig. 3 of Garilli et al. (1996). We also thank M. Fukugita for providing us k-correction data and N. Trentham for the information about the photometry in his articles.

The work on production and cataloguing of DPOSS at Caltech was supported by a generous grant from The Norris Foundation. R. Gal acknowledges a partial support from a NASA Graduate Fellowship. We are also thankful to the POSSII and DPOSS teams for their efforts.

\section{References}

Abell, G. O. 1958, ApJS, 3, 211

Abell, G. O., Corwin, H. G. Jr., \& Olowin, R. P. 1989, ApJS, 70,1

Adami, C., Nichol, R. C., Mazure, A., et al. 1998, A\&A, 334, 765

Andreon, S. 1998, A\&A, 336, 98

Andreon, S., \& Cuillandre, J.-C. 2000, ApJ, in press

Bautz, L. P., \& Morgan, W. W. 1970, ApJ, 162, L149

Bertin, E., \& Dennefeld, M. 1997, A\&A, 317, 43 
Bernstein, G. M., Nichol, R. C., Tyson, J. A., Ulmer, M. P., \& Wittman, D. 1995, AJ, 110, 1507

Binggeli, B., Sandage, A., \& Tammann, G. A. 1985, AJ, 90, 1681

Binggeli, B., Sandage, A., \& Tammann, G. A. 1988, ARA\&A, 26,509

Butcher, H., \& Oemler, A. Jr. 1984, ApJ, 285, 426

Colless, M. 1989, MNRAS, 237, 799

Deutsch, E. W. 1999, AJ, 118, 1882

Drinkwater, M. J., Phillipps, S., Gregg, M.D., et al. 1999, ApJ, 115, 97

Djorgovski, S. G., Gal, R. R., Odewahn, S. C., et al. 1999, in Wide Field Surveys in Cosmology, ed. S. Colombi, Y. Mellier, \& B. Raban

Fukugita, M., Shimasaku, K., \& Ichikawa, T. 1995, PASP, 107, 945

Gaidos, E. J. 1997, AJ, 113, 117

Gal, R. R., de Carvalho, R. R., Odewahn, S. C., \& Djorgovski, S. G. 2000, in preparation

Garilli, B., Bottini, D., Maccagni, D., Carrasco, L., \& Recillas, E. 1996, ApJS, 105, 191

Garilli, B., Maccagni, D., \& Andreon, S. 1999, A\&A, 342, 408 (GMA99)

Huang, J.-S., Cowie, L. L., Gardner, J. P., et al. 1997, ApJ, 476, 12

Jarvis, J. F., \& Tyson, J. A. 1981, AJ, 86, 476

Jerjen, H., \& Tammann, G. A. 1997, A\&A, 321, 713
Jones, L. R., Fong, R., Shanks, T., Ellis, R. S., \& Peterson, B. A. 1991, MNRAS, 249, 481

Lugger, P. M. 1986, ApJ, 303, 535

Koo, D. C., \& Kron, R. G. 1992, ARA\&A, 30, 613

Ostriker, J. P., \& Hausman, M. A. 1977, ApJ, 217, L125

Ostriker, J. P. 1993, ARA\&A, 31, 689

Reid, I. N., Brewer, C., Brucato, R. J., et al. 1991, PASP, 103, 661

Sandage, A., Binggeli, B., \& Tammann, G. 1985, AJ, 90, 1759

Schechter, P. 1976, ApJ, 203, 297

Secker, J., \& Harris, W. E. 1996, ApJ, 496, 623

Thuan, T. X., \& Gunn, J. E. 1976, PASP, 88, 543

Trentham, N. 1997, MNRAS, 290, 334

Trentham, N. 1998, MNRAS, 294, 193

Valotto, C. A., Nicotra, M. A., Muriel, H., \& Lambas, D. G. 1997, ApJ, 479, 90

van Dokkum, P., Franx, M., Kelson, D. D., et al. 1998, ApJ, 500, 714

Wade, R. A., Hoessel, J. G., Elias, J. H., \& Huchra, J. P. 1979, PASP, 91, 35

Weir, N., Fayyad, U. M., \& Djorgovski, S. G., 1995a, AJ, 109, 2401

Weir, N., Djorgovski, S. G., \& Fayyad, U. M. 1995b, AJ, 110, 1

Weir, N., Fayyad, U. M., Djorgovski, S. G., \& Roden, J. 1995c, PASP, 107, 1243

Zwicky, F. 1957, Morphological Astronomy (Springer, Berlin) 